\title{
MALDI In-Source Photooxidation Reactions for Online Peptide Tagging**
}

\author{
Liang Qiao, Christophe Roussel, Jingjing Wan, Jilie Kong, Pengyuan Yang, Hubert H. Girault, \\ and Baohong Liu*
}

As one of the most widely used ionization techniques, MALDI or other related techniques such as surface-enhanced laser desorption ionization can transfer neutral solid-state samples to gas-phase ions for study in proteomics. ${ }^{[1,2]}$ Even if the underlying physicochemical phenomena occurring in these processes are still a matter of debate, the applications have been widely used. ${ }^{[3]}$ Various substrates and matrices have been employed to endow additional functions on the target plates either to assist the ion yield for nonvolatile analytes $^{[4]}$ or to bring about specific interactions with target biomolecules from complex samples. ${ }^{[5,6]}$ Herein, we propose a new concept for driving in-source photoelectrochemical redox reactions under UV laser irradiation and designing a highly porous $\mathrm{TiO}_{2}$ photoelectrode on a steel plate to achieve very efficient electron-transfer reactions with electron donors or acceptors present in the sample or added to the sample.

The photoelectrode is made of $\mathrm{TiO}_{2}$ nanoparticles, similar to that used for the design of dye-sensitized solar cells. ${ }^{[7]}$ Under UV irradiation, the large number of $\mathrm{TiO}_{2}$ nanoparticles absorb photons generating electron-hole pairs and therefore acting as photosensitizers to drive very efficiently electron-transfer reactions. ${ }^{[8]}$ For MALDI-MS analysis, the photoelectrode is coated with the sample and a matrix overlayer ( $\alpha$-cyano-4-hydroxycinnamic acid; CHCA) is added to assist light-energy absorption and protonation of the sample. One advantage of the photoelectrode-modified plate is that it enables the determination of the oxidation or reduction products of a given molecule. If this oxidation/ reduction induces fragmentation of the analytes, the photoelectrode generates in-source decay upon irradiation. Alternatively, the oxidation/reduction products may in turn further react with other molecules, and all the products of these electron-transfer chain reactions can be directly studied by mass spectrometry.

[] L. Qiao, J. Wan, Prof. J. Kong, Prof. P. Yang, Prof. B. H. Liu Department of Chemistry

Fudan University

Shanghai 200433 (China)

Fax: (+ 86) 21-6564-1740

E-mail: bhliu@fudan.edu.cn

Dr. C. Roussel, Prof. H. H. Girault

Laboratoire d'Electrochimie Physique et Analytique

Ecole Polytechnique Fédérale de Lausanne

EPFL, 1015 Lausanne (Switzerland)

[***] This work is supported by NSFC $(20775016,20575013,20525519)$ 973 Program (2007CB714506), Shanghai Leading Academic Discipline B109, and the Swiss-China cooperation research program.

0 Supporting information for this article is available on the WWW under http://www.angewandte.org or from the author.
To illustrate this principle, we use the addition reaction of oxidized hydroquinone on cysteine-containing peptides, which has previously been demonstrated with electrospray ionization. ${ }^{[9,10]}$ Indeed, selective tagging of specific amino acids is an interesting protocol in protein profiling by peptide mass fingerprinting. ${ }^{[11]}$ We show here that a selective and efficient in-source tagging reaction can be developed by combining photoelectrochemical reactions with sample ionization for high-throughput analysis with MALDI-MS. In this case, the unique peptides can be revealed from the digested proteomes. Such a photoinitiated tagging strategy is simple and does not cause sample loss, thus providing powerful information to facilitate accurate and comprehensive protein identification. $^{[12]}$

For this purpose, a photoelectrode array based on $\mathrm{TiO}_{2}$ nanoparticles was fabricated both as a photoreactive substrate and as a porous support to deposit the sample and the matrix on a stainless steel target plate for MALDI analysis (see the Supporting Information). This mesoporous photoelectrode exhibiting a large specific surface area enables a high oxidation capacity, as the photon capture cross-section is very large. Figure 1 shows an illustration of the tagging

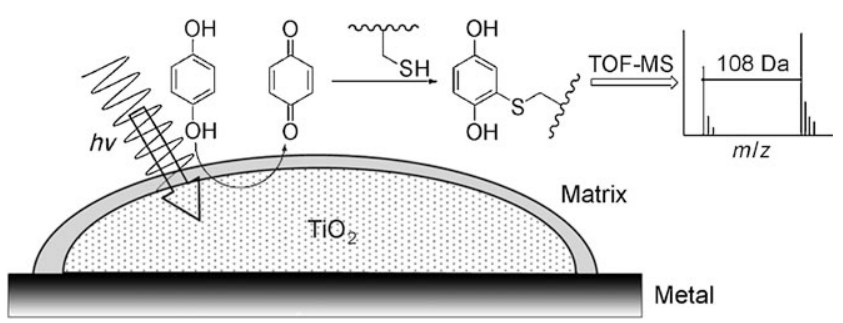

Figure 1. In-source tagging of cysteine-containing peptides.

reaction taking place on the photoelectrode-modified target plate comprising a stainless steel substrate, a photoreactive phase composed of $\mathrm{TiO}_{2}$ nanoparticles, the deposited hydroquinone and cysteine-containing peptides, and the overlayer of the crystalline matrix. Upon irradiation by the UV laser, hydroquinone is photooxidized to benzoquinone, which can in turn react with cysteine-containing peptides to tag them concomitantly with the sample ionization.

To study the photooxidative capacity of the $\mathrm{TiO}_{2}$ photoelectrode for the tagging reaction of cysteine-containing peptides, SSDQFRPDDCT (C-pep) was chosen as a model peptide. At a C-pep/hydroquinone molar ratio of 1:1 (1.5 pmol each), a signal situated at $\mathrm{m} / \mathrm{z}$ 1378.6 was observed, corresponding to the tagged C-pep peptide in addition to the 
native C-pep at $m / z 1270.5$ (Figure 2 b). This result can be compared to the MS response obtained on a regular target plate without $\mathrm{TiO}_{2}$ nanoparticle modification, with which no
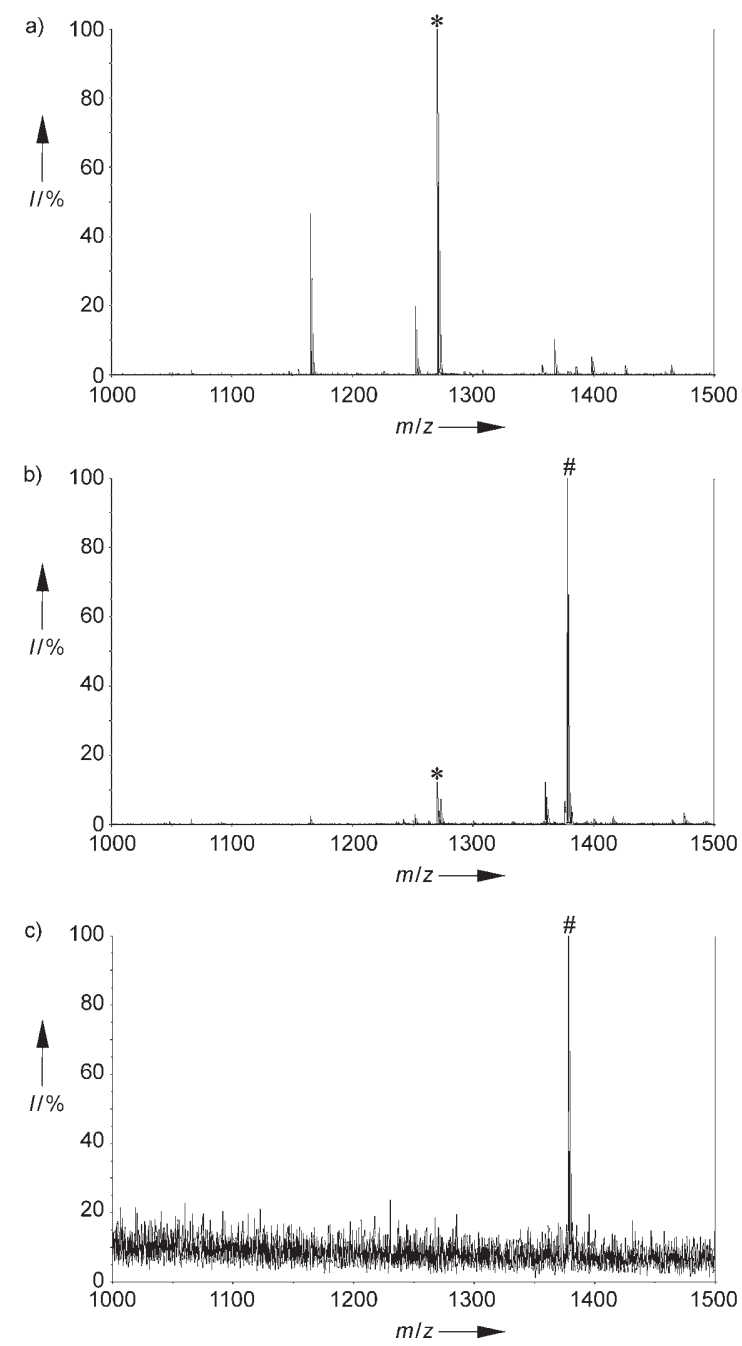

Figure 2. Mass spectrum of C-pep/hydroquinone (1:1, 1.5 pmol each) performed on a blank target plate (a) and a $\mathrm{TiO}_{2}$-modified target plate (b), and mass spectrum of C-pep/hydroquinone (1:1, $7 \mathrm{fmol}$ each) performed on the $\mathrm{TiO}_{2}$-modified target plate (c); *: protonated form of native C-pep ( $\mathrm{m} / \mathrm{z}$ 1270.5); \#: protonated form of tagged C-pep ( $\mathrm{m} / \mathrm{z}$ 1378.6).

tagged peptide signal can be detected with the same sample (Figure 2a). The detection limit of this tagging strategy is at the femtomole level (Figure $2 \mathrm{c}$ ). The tagging information was investigated by using a positive-ion MS/MS method on the ions of both tagged and untagged peptides. The result clearly shows that the hydroquinone moiety is linked on the cysteine residue (Figure S1 and Table S1 in the Supporting Information), which supports that the oxidized hydroquinone molecule undergoes an addition reaction with the sulfhydryl groups on the C-pep peptide. We can also see that the tags yielded by this in-source addition reaction are stable during the collision-induced dissociation process, which is of great value for chemical tagging strategies. The selectivity of the photoinduced cysteine labeling was further confirmed by using cysteine-free peptides containing all the other natural amino acids, that is, SSDQFRPDDGT, AEKTKEGVW, RPVKVYPNGAEDESAEAFPLEF, DRVYIHPF, and DNEAYEMPSEE. With the same conditions, no tagged peak was observed (Figure S2a-e in the Supporting Information), thus indicating that the tagging reaction is highly specific to cysteine residues even if other nucleophilic amino acids are present in the sample.

However, the exact oxidation mechanism of the in-source tagging reaction is likely to be complex. Considering the amount of matrix material deposited on the porous photoelectrode, the matrix overlayer thickness is about $0.5 \mu \mathrm{m}$, which represents about $7 \times 10^{14}$ matrix molecules per $\mathrm{mm}^{2}$, which is less than the laser fluence of $10^{15}$ photons per $\mathrm{mm}^{2}$ per laser pulse. Thus, although there is a matrix overlayer that strongly absorbs the incoming laser light, part of the radiation can be transmitted to the $\mathrm{TiO}_{2}$ surface and induces the photooxidation tagging reaction (see the Supporting Information). The $\mathrm{TiO}_{2}$ nanoparticles acting here as photosensitizers ${ }^{[13,14]}$ are very strong oxidants able to oxidize hydroquinone either directly or through photogenerated reactive oxygen species from the matrix, and the subsequent addition reaction takes place in the ion source. On the photoelectrode-modified plate, the redox reactions also involve proton transfer, and here the acid acts as an efficient proton donor and hole conductor, thereby favoring the longdistance oxidation reaction. Moreover, the substituted hydroquinones 2,5-dihydroxybenzoic acid (DHB) and 2-methoxyhydroquinone (MOHQ) were employed to study the reaction activity. The results show that the tagging occurs also with those two compounds and that the ratios of peak intensities of tagged/untagged peptide are found to follow the order of $\mathrm{MOHQ}>\mathrm{HQ}>\mathrm{DHB}$ at a peptide/quinone ratio of $1: 1$ (Figure S3a-c in the Supporting Information). In this case, the presence of the methoxy group on the hydroquinone species (in MOHQ) makes the tagging very efficient.

Furthermore, the tagging reaction was applied to a peptide containing three cysteine residues (ACKCTCM; 3C-pep). As shown in Figure $3 \mathrm{a}$, the signals coming from singly, doubly, and triply tagged peptides can all be observed in one spectrum, together with the untagged peptides. The results show that this highly selective photoelectrochemical tagging strategy provides a convenient route for counting free cysteine residues present in peptides, thus offering valuable supplementary information in the process of database interrogation for protein identification. ${ }^{[15]}$ To illustrate this application, the in-source reaction was employed to specifically recognize cysteine-containing peptides from the complex tryptic digests of $\beta$-lactoglobulin $\mathrm{A}$ and albumin. For $\beta$ lactoglobulin $\mathrm{A}$, four cysteine-containing peptides were revealed (Figure $3 \mathrm{~b}$ ), corresponding to all five cysteine residues present in this protein (Table S2 in the Supporting Information). Considering the information on cysteine content, $\beta$-lactoglobulin A was identified with a database searching score of 196, whereas only 151 was obtained without this information (Figure $\mathrm{S} 4 \mathrm{a}$ in the Supporting Information). Similarly, 19 cysteine-containing peptides were identified from the albumin digest mixture, including one three- 

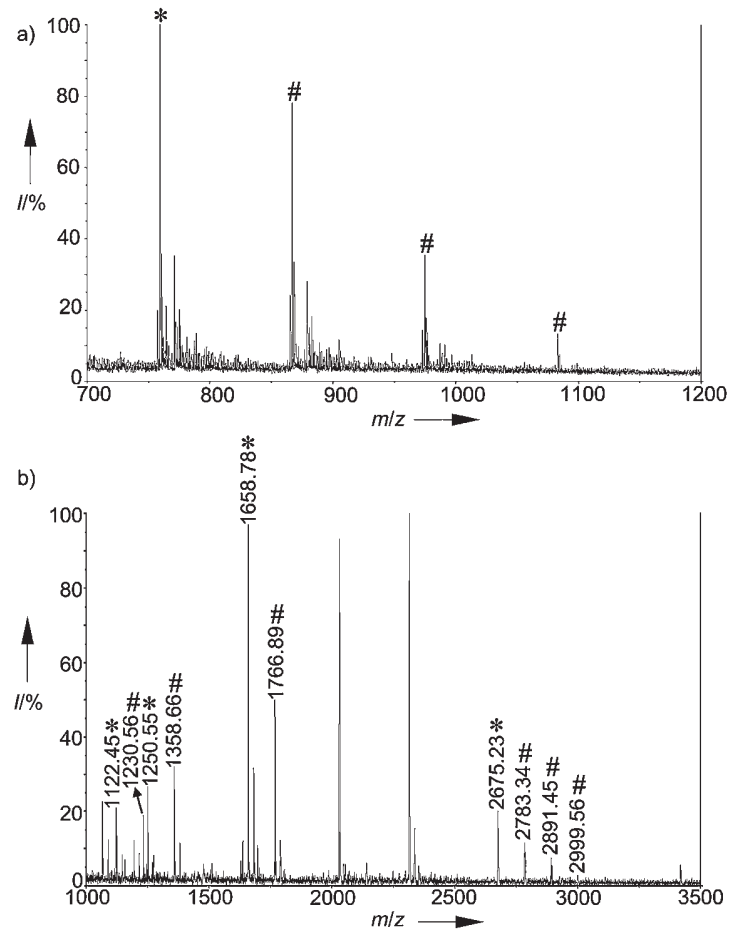

Figure 3. a) Mass spectra of $3 \mathrm{C}$-pep/hydroquinone $(1: 1,1.5 \mathrm{pmol}$ each) on a $\mathrm{TiO}_{2}$-modified target plate; *: protonated form of native $3 \mathrm{C}$ peptide $(\mathrm{m} / \mathrm{z} 759.3)$; \#: singly, doubly, and triply tagged 3C-peptide peaks ( $m / z 867.3,975.3,1083.4)$. b) Mass spectrum of $\beta$-lactoglobulin A digest (ca. $5 \mathrm{pmol}$ ) in the presence of $5 \mathrm{nmol}$ hydroquinone on a $\mathrm{TiO}_{2}$-modified plate; *: protonated form of precursor peptides; \#: protonated form of tagged peptides.

cysteine-, four two-cysteine-, and 14 single-cysteine-containing peptides, resulting in 20 out of 35 cysteine residues in albumin (Table S3 and Figure S5 in the Supporting Information). With the help of this additional information, the database searching score was enhanced from 261 to 424 (Figure S4b in the Supporting Information).

In summary, we have demonstrated that a $\mathrm{TiO}_{2}$ photoelectrode array based plate can be used to photooxidize very efficiently target molecules such as hydroquinone during laser desorption ionization MS analysis. In particular, this approach can be conveniently used for the in-source tagging of cysteine residues in a given peptide. With this strategy, we can identify the unique peptides from a complex peptide mixture as well as count the number of cysteine residues. This in-source photoreactive protocol can facilitate the high-throughput study of metabolic pathways in biological processes.

\section{Experimental Section}

A mixture $(0.4 \mu \mathrm{L})$ of C-pep/hydroquinone (1:1), 3C-pep/hydroquinone (1:1), C-pep/DHB (1:1), C-pep/MOHQ (1:1), or protein digest/ hydroquinone was deposited on the $\mathrm{TiO}_{2}$ photoelectrode array immediately after mixing and dried at room temperature in the dark for 10 min. After drying, CHCA $(0.4 \mu \mathrm{L})$ was dropped onto the array and then dried under the same conditions for $5 \mathrm{~min}$ and finally subjected to MALDI-TOF-MS (Applied Biosystems 4700 Proteomics Analyzer, 2000 laser shots $(355 \mathrm{~nm}, 200 \mathrm{~Hz})$ at a laser intensity of 5500 instrument units). As a comparison, C-pep/hydroquinone (1:1; $0.4 \mu \mathrm{L})$ was dropped onto a blank plate, dried under the same conditions, and a CHCA matrix $(0.4 \mu \mathrm{L})$ was then dropped onto the plate, dried (same conditions), and subjected to MALDI-TOF-MS. All the peptides and hydroquinone were dissolved in deionized water.

Received: August 23, 2007

Revised: November 6, 2007

Published online: February 25, 2008

Keywords: mass spectrometry - peptides - photoelectrodes . photooxidation - titanium dioxide

[1] R. Aebersold, M. Mann, Nature 2003, 422, 198-207.

[2] R. Knochenmuss, Analyst 2006, 131, 966-986.

[3] G. L. Nelsestuen, Y. Zhang, M. B. Martinez, N. S. Key, B. Jilma, M. Verneris, A. Sinaiko, R. S. Kasthuri, Proteomics 2005, 5, $4012-4024$

[4] J. Wei, J. M. Buriak, G. Siuzdak, Nature 1999, 399, 243-246.

[5] R. W. Nelson, D. Nedelkov, K. A. Tubbs, Anal. Chem. 2000, 72, 404A-411A.

[6] G. R. Blacken, M. Volny, T. Vaisar, M. Sadilek, F. Turecek, Anal. Chem. 2007, 79, 5449-5456.

[7] B. O'Regan, M. Grätzel, Nature 1991, 353, 737-740.

[8] M. Ni, M. K. H. Leung, D. Y. C. Leung, K. Sumathy, Renewable Sustainable Energy Rev. 2007, 11, 401-425.

[9] C. Roussel, L. Dayon, N. Lion, T. C. Rohner, J. Josserand, J. S. Rossier, H. Jensen, H. H. Girault, J. Am. Soc. Mass Spectrom. 2004, 15, 1767-1779.

[10] C. Roussel, L. Dayon, H. Jensen, H. H. Girault, J. Electroanal. Chem. 2004, 570, 187-199.

[11] S. P. Gygi, B. Rist, S. A. Gerber, F. Turecek, M. H. Gelb, R. Aebersold, Nat. Biotechnol. 1999, 17, 994-999.

[12] L. Dayon, C. Roussel, M. Prudent, N. Lion, H. H. Girault, Electrophoresis 2005, 26, 238-247.

[13] O. Carp, C. L. Huisman, A. Reller, Prog. Solid State Chem. 2004, $32,33-177$.

[14] J. F. Liu, C. Roussel, G. Lagger, P. Tacchini, H. H. Girault, Anal. Chem. 2005, 77, 7687-7694.

[15] S. Sechi, B. T. Chait, Anal. Chem. 1998, 70, 5150-5158. 